\title{
A novel classifier model for mass classification using BI-RADS category in ultrasound images based on Type-2 fuzzy inference system
}

\author{
ESMA UZUNHISARCIKLI ${ }^{1, *}$ and VOLKAN GOREKE ${ }^{2}$ \\ ${ }^{1}$ Kayseri Vocational College, Erciyes University, Kayseri, Turkey \\ ${ }^{2}$ Kangal Vocational College, Cumhuriyet University, Sivas, Turkey \\ e-mail: uzunhise@erciyes.edu.tr
}

MS received 1 October 2017; revised 31 December 2017; accepted 1 January 2018; published online 13 July 2018

\begin{abstract}
Ultrasound imaging is an imaging technique for early detection of breast cancer. Breast Imaging Reporting and Data System (BI-RADS) lexicon, developed by The American College of Radiology, provides a standard for expert doctors to interpret the ultrasound images of breast cancer. This standard describes the features to classify the tumour as benign or malignant and it also categorizes the biopsy requirement as a percentage. Biopsy is an invasive method that doctors use for diagnosis of breast cancer. Computer-aided detection (CAD)/diagnosis systems that are designed to include the feature standards used in benign/malignant classification help the doctors in diagnosis but they do not provide enough information about the BI-RADS category of the mass. These systems classify the benign tumours with $90 \%$ biopsy possibility (BI-RADS-4) and with $2 \%$ biopsy possibility (BI-RADS-2) in the same category. There are some studies in the literature that make category classification via commonly used classifier methods but their success rates are low. In this study, a twolayer, high-success-rate classifier model based on Type-2 fuzzy inference is developed, which classifies the tumour as benign or malignant with its BI-RADS category by incorporating the opinions of the expert doctors. A 99.34\% success rate in benign/malignant classification and a $92 \%$ success rate in category classification (BIRADS 2,3,4,5) were obtained in the accuracy tests. These results indicate that the CAD system is valuable as a means of providing a second diagnostic opinion when radiologists carry out mass diagnosis.
\end{abstract}

Keywords. Ultrasound; CAD; Type-2 fuzzy.

\section{Introduction}

According to recent research, $14.79 \%$ of deaths caused by cancer are due to breast cancer [1]. Breast cancer is defined as the abnormal cell growth around the breast and it is classified into two classes as benign or malignant [2]. Mammography and ultrasound imaging are the most principal imaging techniques $[3,4]$. With respect to the ultrasound imaging, mammograms cause the doctors to make much more incorrect detections, meaning a more falsepositive detection rate [5]. Breast Imaging Reporting and Data System (BI-RADS) lexicon, which is developed by The American College of Radiology for the diagnosis of sonographic lesions, includes the description of mass features such as shape, margin and orientation. Predictive values of these features were examined by a study by Hong in 2005 . According to this study, predictive values for malignant masses are spiculated margins $86 \%$ and irregular shape $62 \%$, while the predictive values for benign masses are circumscribed margins $90 \%$ and shape $84 \%$ [6, 7]. BI-RADS categorizes the lesions with respect to the probability of biopsy

*For correspondence requirement. These categories are Category $20 \%$, Category 3 is below 2\%, Category 4 is 3\%-94\% and Category 5 is 95\% [8]. The most basic standard to determine whether the tumour is malignant or not is through biopsy. But more than $70 \%$ of the results obtained via biopsy (which is an invasive method) are benign [9]. Redundant biopsies both increase the cost and cause emotional pressure on the patient [10]. In the case of lesion characterization, complex anatomy, variation in the presentation of benign and malignant states and the varying abilities of the physicians may result in interpretation errors. Computer-aided detection (CAD) systems have more space in modern medicine, providing specialists with a second source of information and increasing the diagnostic success rates. These systems have been implemented to support the detection and characterization of breast lesions from digital mammography and ultrasound images, to assist the diagnosis of lung cancer using chest radiographs and Computed Tomography images, to distinguish Tourette's syndrome from chronic tic disorder based on Single Photon Emission Computed Tomography brain imaging and to support the diagnosis of functional brain disorders using Positron Emission Tomography images. CAD systems that help the doctor in the interpretation of 
Table 1. Survey of CAD systems.

\begin{tabular}{|c|c|c|c|c|c|c|}
\hline Reference no. & Size of data set & Classifier type & Accuracy $(\%)$ & Features & nof & Feature selection \\
\hline [13] & 49 (32 B/17 M) & DA function & 87.80 & Tx. \& Mh. & 5 & No \\
\hline [20] & $250(215 \mathrm{~B} / 35 \mathrm{M})$ & SVM & 94.40 & Tx. & 2 & No \\
\hline [28] & $112(54 \mathrm{~B} / 58 \mathrm{M})$ & SVM & 91.10 & Tx. \& Mh. & 85 & PCA \\
\hline [29] & $210(120 \mathrm{~B} / 90 \mathrm{M})$ & SVM & 90.95 & Mh. & 6 & No \\
\hline [30] & $78(26 \mathrm{~B} / 52 \mathrm{M})$ & SVM & 95.70 & Tx. \& Mh. & 10 & PCA \\
\hline [31] & $148(119 \mathrm{~B} / 29 \mathrm{M})$ & SVM & 85.14 & Tx. \& Mh. & 14 & GA \\
\hline [32] & $200(102 \mathrm{~B} / 98 \mathrm{M})$ & Biclustering + BPNN & 94.00 & Mh. & 19 & Variance metric \\
\hline [33] & $178(88 \mathrm{~B} / 90 \mathrm{M})$ & FCM & 95.86 & Tx. \& Mh. & 457 & Filter \\
\hline [33] & $178(88 \mathrm{~B} / 90 \mathrm{M})$ & BPNN & 94.14 & Tx. \& Mh. & 19 & Filter \\
\hline [34] & $118(84 \mathrm{~B} / 34 \mathrm{M})$ & SVM & 82.20 & Mh. & 19 & PCA \\
\hline [35] & $210(120 \mathrm{~B} / 90 \mathrm{M})$ & $\mathrm{SVM}+\mathrm{GA}$ & 95.24 & Tx. \& Mh. & 6 & GA \\
\hline [36] & $321(113 \mathrm{~B} / 208 \mathrm{M})$ & SVM & 86.92 & Tx. \& Mh. & 36 & By radiologists \\
\hline [36] & $321(113 \mathrm{~B} / 208 \mathrm{M})$ & ANN & 86.60 & Tx. \& Mh. & 36 & By radiologists \\
\hline [36] & $321(113 \mathrm{~B} / 208 \mathrm{M})$ & KNN & 83.80 & Tx. \& Mh. & 36 & By radiologists \\
\hline [84] & $65(31 \mathrm{~B} / 34 \mathrm{M})$ & FCMNN & 92.00 & Tx. & 49 & No \\
\hline [38] & $178(88 \mathrm{~B} / 90 \mathrm{M})$ & SVM & 94.40 & Tx. \& Mh. & 457 & Filter \\
\hline [38] & $178(88 \mathrm{~B} / 90 \mathrm{M})$ & BPNN & 96.60 & Tx. \& Mh. & 19 & Filter \\
\hline [72] & $244(166 \mathrm{~B} / 78 \mathrm{M})$ & Decision tree & 89.00 & Tx. \& Mh. & 6 & Statistical method \\
\hline
\end{tabular}

Abbreviations: Tx. - texture, Mh. - morphologic, B - benign, M - malignant, GA - genetic algorithm, DA - discriminant analysis, FCMNN - Fuzzy Cerebellar Model Neural Networks, BPNN - Back-Propagation Neural Network, FCM - fuzzy C-Mean clustering, ANN - Artificial Neural Network, PCA - principal component analysis, nof - total number of features

radiologic images also decrease the detection time [11, 12]. A typical CAD system architecture consists of four main modules, namely image pre-processing, definition of region of interest (ROI), feature extraction/selection from ROI and classification of ROI [11, 13]. In this work, the mass areas in 153 breast ultrasound images that belong to the women patients were marked by an expert radiologist. The smallest rectangular area covering the tumour was cut off from the image to be used as the ROI. The speckle noise in the subimages was reduced by filtering and image enhancement. Medical information that the expert doctor uses while determining the BI-RADS category and the type of mass were noted during the interpretation of each image. A CAD model based on the Type-2 fuzzy inference was designed using this information and the feature values were obtained using the ROI. Depending also on the expert opinion, the proposed model is based on a novel two-layer Type-2 fuzzy inference system that classifies the tumour area as benign or malignant with its BI-RADS category. The rest of the paper is as follows. In section 2, there is a literature review. In section 3, the fuzzy inference system is examined. In section 4, the details of the proposed CAD model are provided. In section 5 , the classification success-rate tests of the proposed model are presented. Finally, in section 6, the results and the future work are discussed.

\section{Literature review}

In recent years, classification of breast tumours in ultrasound images is a hot research point. Many CAD systems have been proposed to distinguish the benign mass from the malignant mass in these studies. A survey on the research work done on such CAD systems is summarized in table 1 . Columns of table 1 present the size of the data set used in the study, type of the classifier, the success rate of the classifier, the type of the features used in the study, the size of the total features used in the study and feature selection methods if there are any. The used abbreviations are summarized in the last row of table 1 .

\subsection{Image preprocessing}

The most severe disadvantage of the ultrasound is the low quality of the image. It affects the precision of the diagnosis negatively [14]. The sound wave used in ultrasound imaging has to pass through the heterogeneous tissue with different acoustic properties before it reaches the converter. This situation causes distortion in the image called the speckle noise [15]. Many filtering architectures have been proposed to remove the speckle noise $[16,17]$.

\subsection{Obtaining ROI}

ROI is the area that includes the mass and is used to extract the features [9]. There are two methods to obtain the ROI. One of these is obtaining automatically by segmentation methods [9, 18, 19] and the other is manually by expert radiologist marking [20, 21]. Using the ROI, various feature sets grouped as texture, morphological, model-based and descriptor are obtained, which can be used to distinguish the breast cancer lesions as benign and malignant $[10,22]$. 


\subsection{Texture features extraction}

Texture features that are very commonly used in literature can be obtained using first-order or second-order statistical calculations such as grey-level co-occurrence matrix (GLCM) [21, 23]. GLCM matrices are obtained by calculating the joint probability density functions of the pixel pairs at a specific orientation and distance in the image matrix [24]. Let $i, j(i, j=(0,1,2, \ldots, N-1)$ represent the grey levels of two pixels and let $\mu$ be the relationship of the position between two pixels as orientation and distance. Then $P_{\mu}(i, j)$ represents the GLCM [25]. Texture features can be extracted from a grey-scale image using GLCM [26]. Texture features are given in Eqs. (1)-(4):

$$
\begin{gathered}
\text { energy }=\sum_{i=0}^{N-1} \sum_{j=0}^{N-1} P_{\mu}^{2}(i, j) \\
\text { contrast }=\sum_{t=0}^{N-1} t^{2}\left\{\sum_{i=0}^{N-1} \sum_{j=0}^{N-1} P_{\mu}(i, j)\right\} \\
\text { correlation }=\frac{\sum_{i=0}^{N-1} \sum_{j=0}^{N-1} P_{\mu}(i, j) i j-m_{1} m_{2}}{\sigma_{1}^{2} \sigma_{2}^{2}} \\
\text { homogeneity }=\frac{\sum_{i=0}^{N-1} \sum_{j=0}^{N-1} P_{\mu}(i, j)}{1+(i+j)^{2}}
\end{gathered}
$$

$m_{1}, m_{2}, \sigma_{1}$ and $\sigma_{2}$ are defined, respectively, by Eqs. (5), (6), (7) and (8):

$$
\begin{gathered}
m_{1}=\sum_{i=0}^{N-1} i \sum_{j=0}^{N-1} P \mu(i, j), \\
m_{2}=\sum_{i=0}^{N-1} j \sum_{j=0}^{N-1} P \mu(i, j), \\
\sigma_{1}^{2}=\sum_{i=0}^{N-1}\left(1-\mu_{1}\right)^{2} \sum_{j=0}^{N-1} P \mu(i, j), \\
\sigma_{2}^{2}=\sum_{j=0}^{N-1}\left(1-\mu_{2}\right)^{2} \sum_{i=0}^{N-1} P \mu(i, j) .
\end{gathered}
$$

The histogram of the grey levels is an abstract of the statistical information that is present in the image [27]. A grey-scale image, represented as a two-dimensional matrix mathematically, can be defined as a function $f(x, y)$ where $x=1, \ldots, N-1$ represents the row index and $y=1, \ldots, M-1$ represents the column index. Let $G$ be the total number of grey-scale numbers; then the function $f(x, y)$ can take $i=1, \ldots, G-1$ discrete values. Grey-scale histogram shows the number of the pixels that have the same intensity value in the image and given by Eq. (9):

$$
h(i)=\sum_{x=0}^{N-1} \sum_{y=0}^{M-1} \delta(f(x, y), i) .
$$

Here $\delta(j, i)$ is the Kronecker delta function and given by Eq. (10):

$$
\delta(j, i)=\left\{\begin{array}{l}
1 \text { if } j=i \\
0 \text { if } j \neq i
\end{array}\right.
$$

In Eq. (11) the probability density of the recurrence of grey level can be obtained by dividing $h(i)$ by the number of pixels in the image:

$$
p(i)=h(i) / N M, \quad i=0, \ldots, G-1 .
$$

Equations (12)-(15) show that first-order texture features can be obtained using $p(i)$ :

$$
\begin{gathered}
\text { mean } \mu=\sum_{i=0}^{G-1} i P(i), \\
\text { variance } \sigma^{2}=\sum_{i=0}^{G-1}(i-\mu)^{2} P(i), \\
\text { standard deviation } \sigma=\sqrt{\sum_{i=0}^{G-1}(i-\mu)^{2} P(i),} \\
\text { kurtosis } k=\sigma^{-4} \sum_{i=0}^{G-1}(i-\mu)^{4} P(i)-3 .
\end{gathered}
$$

Other than the texture features obtained conventionally from the smallest area covering the tumour (ROI), Cheng et al [10] have obtained texture-based features that also include a morphological feature (size) besides the texture information using together the number of lateral/depth pixels and grey-level values of ROI. Amin et al [28] divided the smallest rectangular area enclosing the tumour into 9 images and gathered the first-order texture features obtained from each sub-image into the sets called vertical, horizontal and central orientation. They also obtained a new texture-based feature set by dividing the texture feature values obtained from the whole ROI by the values obtained from these sets [28].

\subsection{Morphological (shape, margin) features extraction}

In lots of CAD systems, texture and morphological features [29] have been used together to increase the classification success rate [13, 28, 30-37]. Morphological features are independent of various ultrasound systems or machines in the diagnosis of breast cancer masses. Therefore, it is more advantageous to use morphological features in CAD systems [38] where they can also define the margin and shape 
of the mass [39, 40]. The most common morphological features used in CAD systems are roundness, perimeter, area, area perimeter ratio, long-axis-short-axis ratio and most importantly the spiculation [34, 40-42]. Roundness is defined as the shape feature that represents the roundness of the mass shape in BI-RADS lexicon and is given by Eq. (16) [34]. A high value of roundness increases the possibility of malignancy [43]:

$$
\text { roundness }=4(\text { area }) / \pi\left(\text { max_diameter }{ }^{2}\right) .
$$

Here, the max_diameter is the length of the long axis of the equivalent ellipsis that covers the mass. Area is the area in pixels bounded by the contour of the shape that forms the mass. Malignant masses usually have a larger area than the benign ones [28, 42]. Perimeter is the measure of the mass periphery. Malignant masses have irregular shape and a higher perimeter value [44, 45]. Ap_ratio feature is calculated by dividing the mass area by the perimeter of the tumour and is given by Eq. (17):

$$
\text { Ap_ratio }=\text { area } / \text { perimeter } .
$$

Las_ratio is the ratio of the long axis (in pixels) to the short axis (in pixels) of the shape that forms the tumour and is given in Eq. (18). The ratio of the long axis to the short axis is an important parameter in segregation of the malignant and benign tumours. The elliptic shape of a mass is a significant feature for the mass to be classified as benign [46].

$$
\text { Las_ratio }=\text { long-axis length/short-axis length. }
$$

Spiculations are spiked contour gaps and lines out of the mass (arrows). Spiculations are the highest BI-RADS predictive value morphological features for the classification of malignancy $[43,47]$. Different methods have been proposed for the calculation of spiculation. Among these methods, SI value has been calculated for every curved part of each of the mass border as the ratio of the length of the contour to the width of the floor [48]. One of the most promising methods for the description of spiculation depends on the Fourier descriptors of the image contour [49]. Here, the polar coordinates and the mass centre of the contour pixels of the mass were obtained and the radial distance was calculated. Fourier transform of the radial distance was obtained and the sum of the high-frequency components with respect to the cut-off frequency pi/4 was divided by the sum of the low-frequency components; then this ratio was used as a feature [50]. In another study, the skew angle from each pixel, at the contour of the mass to the convenient pixels, was used to specify the spiculation feature. Spiculated regions have a lower skew angle (45$60^{\circ}$ ) [43]. In another method, the average distance between a virtual circular contour (with its centre overlapping the centre of the mass and having the same area as that of the mass) and the pixels of $N$ tumour contours outside this circular contour was calculated and a feature was obtained to measure the spiculation [39].

\subsection{Feature selection}

Many features can be extracted from ultrasound images. To correctly classify and diagnose the benign and malignant masses in the breast cancer data set, it is very important to select the features in an optimal way [51]. There are many feature selection systems in the literature. They are principal component analysis (PCA) [30, 44], artificial intelligence algorithms [31], bidirectional search [29], $t$-test for selecting class-descriptor features and statistical significance tests such as Wilcoxon-Mann-Whitney (WMW) test [52] and backward feature elimination method [53].

\subsection{Classification}

The most common classifiers used in the classification of tumours in the ultrasound images as benign or malignant are summarized in table 1. Besides, machine learning methods such as artificial neural networks (with different topologies: multilayer perceptron, probabilistic neural network) $[45,52]$, fuzzy SVM [5] and $C$ means (to cluster the data to $C$ clusters using the least squares method) [54], statistical analysis methods (chi-square and Mann-Whitney $U$ test, logistic regression classifier, Fisher's exact test, $t$ test) $[3,55], k$ nearest neighbourhood (KNN) algorithm (classifies the test samples based on the $k$ nearest training samples in the feature space) and citation KNN [56], which is an enhanced KNN algorithm, were also used as classifiers.

\section{Fuzzy inference systems}

Fuzzy set theory, which was proposed by Zadeh in 1965 , deals with the vagueness of the information. It is less restrictive than the classical set theory and more convenient for the processing of data provided by humans. The membership or non-membership (0 or 1$)$ in the classical set theory is mapped onto the continuous domain $(0,1)$ [57]. Fuzzy inference is the mapping of the given inputs to the outputs via an inference mechanism using if-then rules, membership functions and fuzzy logic. Among the fuzzy inference models, the well-known Mamdani model uses the semantic rules, union and intersection operators ( $\max -\min )$ [58]. This model consists of fuzzification, if-then linguistic rules and defuzzification steps. The fuzzification maps the crisp input values to the corresponding membership values using the triangular, Gaussian and trapezoidal membership functions. The histogram of the given data set is one of the methods to choose the membership function [59]. If-then rules of Mamdani algorithm are given in Eq. (19):

$$
R_{i} \text { : if } x_{i} \text { is } A_{i 1} \text { and...then } y_{i} \text { is } B_{i}(\text { for } i=1,2,3, \ldots, k) \text {. }
$$

Here $k$ is the number of rules, $x$ is input variable (antecedent value) and $y$ is output value (consequent value). 
The basic form of a fuzzy composition processing is $B=$ $A \circ R$. Here $A$ is the antecedent defined in the $X$ universe. $B$ is the consequent defined in the $Y$ universe. $R$ is the fuzzy relation between $X$ and $Y$. The first step in Mamdani-type fuzzy inference system is the calculation of the degree of fulfilment $\alpha$ for each rule (i) using the membership degrees $\mu$. It is given by Eq. (20) [60]:

$$
\alpha_{i}=\mu_{A i 1}\left(X_{1}\right) \wedge \mu_{A i 2}\left(X_{2}\right) \wedge \ldots \wedge \mu_{A i n}\left(X_{n}\right) .
$$

In the second step, the fuzzy output set $B_{i}$ is derived for each rule using the $t$-norm. It is given by Eq. (21):

$$
\mu_{B i}(y)=\alpha_{i} \wedge \mu_{B i}(y) .
$$

In the third step the output fuzzy sets are aggregated using the maximum. It is given by Eq. (22):

$$
\mu_{B}=\max \left(\mu_{B i}(y)\right), \quad i=1,2, \ldots, k .
$$

In the final step the output fuzzy set is converted to the crisp value via defuzzification. One of the methods for defuzzification is the centre of gravity (COG). It is given by Eq. (23):

$$
y=\int B(y) y d y / \int B(y) d y .
$$

\subsection{Type-2 fuzzy inference system}

Zadeh proposed the Type-2 fuzzy sets in 1975. Therefore, the fuzzy set that he proposed earlier was called Type 1 [61]. A fuzzy logic system (FLS) using at least one Type-2 fuzzy set is called a Type-2 FLS. The structure of the Type2 fuzzy rules is the same as that of the Type- 1 fuzzy rules except for the structure of the membership functions. Unlike Type 1, in the output process where the crisp value is calculated, it also includes the type reduction unit [62]. The membership value of each element in the Type-1 fuzzy set is described with crisp values in $[0,1]$ while the membership value of each element in the Type-2 fuzzy set is described as fuzzy sets. Type- 1 fuzzy set with $n$ elements is defined with $n$ fuzzy sets with a membership value of [0, 1], where $n$ fuzzy sets generate the footprint of vagueness (FOU) [59]. The recent studies on Type-2 fuzzy sets depend on the definition by Mendel [63-65]. According to this definition, instead of using the $n$ fuzzy sets that constitute the FOU, using only the lower and upper membership functions (LMF, UMF) simplifies in obtaining the result. Therefore, the union of lower and upper membership functions is described as FOU [66]. There are various artificial intelligence optimization algorithms in the literature such as genetic algorithms [67]. The equation of $F O U$ is given in Eq. (24), while the membership functions are shown in figure 1:

$$
\operatorname{FOU}(\hat{A})=\cup\left[\bar{\mu}_{\hat{A}}(x), \quad \underline{\mu}_{\hat{A}}(x)\right] / x \quad \forall x \in X .
$$

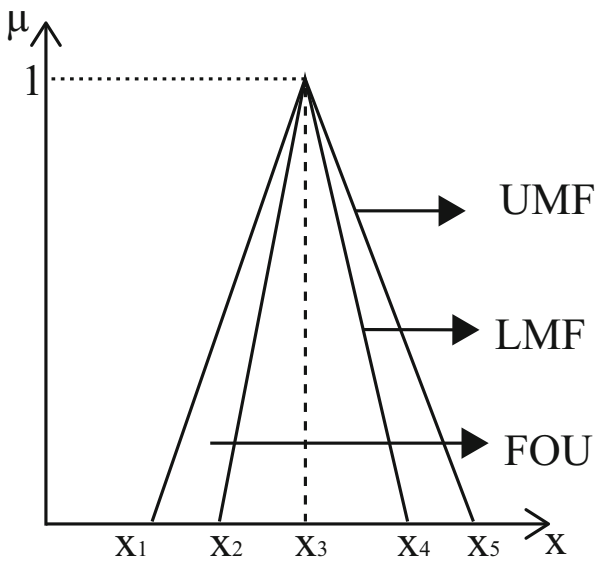

Figure 1. Type-2 triangular membership function (UMF - upper membership function, LMF - lower membership function, FOU footprint of uncertainty).

\section{Proposed method}

In this study, a total of 153 ultrasound images containing 64 benign and 89 malignant masses were used (CMSDH K, SSKM Hospital Kolkata and the webpage: http://www. ultrasoundcases.info/About-Us.aspx by Dr T S A Geertsma, The Netherlands). The proposed CAD system for the classification of ultrasound images consists of the following steps.

\subsection{Selection of ROI}

A software has been developed for an expert radiologist to mark the area containing the mass. The software also provides the pixel-based coordinate information of the marked mass contour. Using the coordinate information, the smallest rectangular area is cut off and the ROI image to extract the features has been obtained. As an example, figures 2 and 3 show, respectively, the marked area and the ROI image.

\subsection{Speckle noise reduction and image enhancement}

One of the most common methods to reduce the speckle noise is filtering. In this study, Gaussian filter has been used for the reduction of speckle noise reduction $[16,17]$. Histogram equalization, which is used for enhancing the low visibility of an image, makes the output histogram to a uniform distribution. Contrast limited adaptive histogram equalization is another version of histogram equalization which has different equalization values for each region [68]. In this study, image enhancement has been realized using 


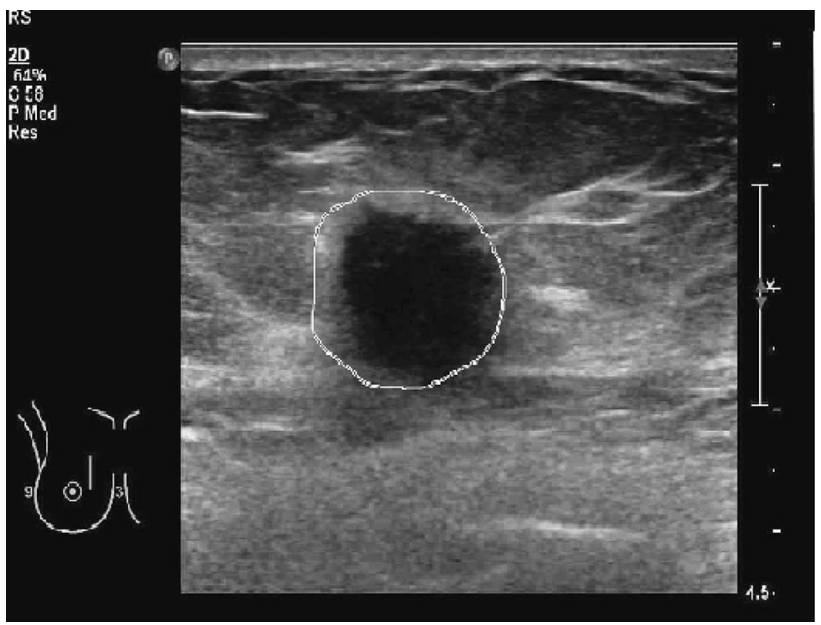

Figure 2. Area marked by a physician.

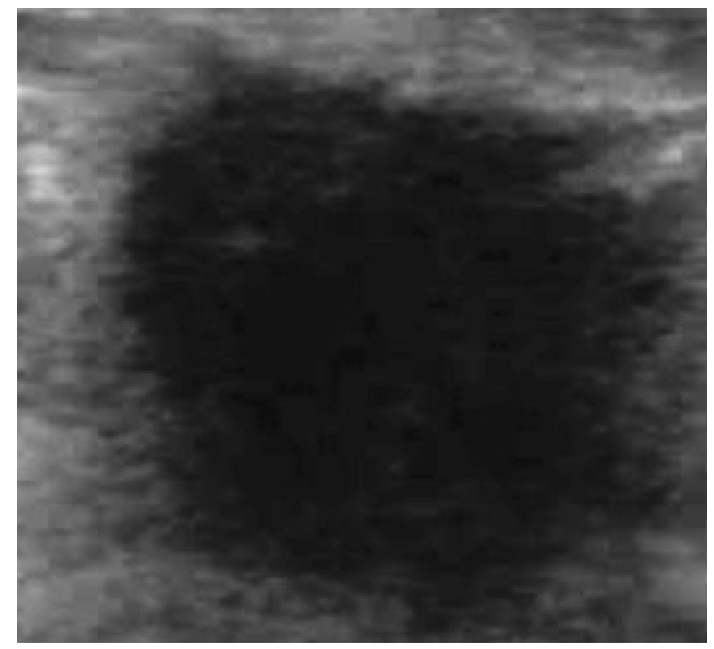

Figure 3. ROI image of malignant case segmented from BUS image.

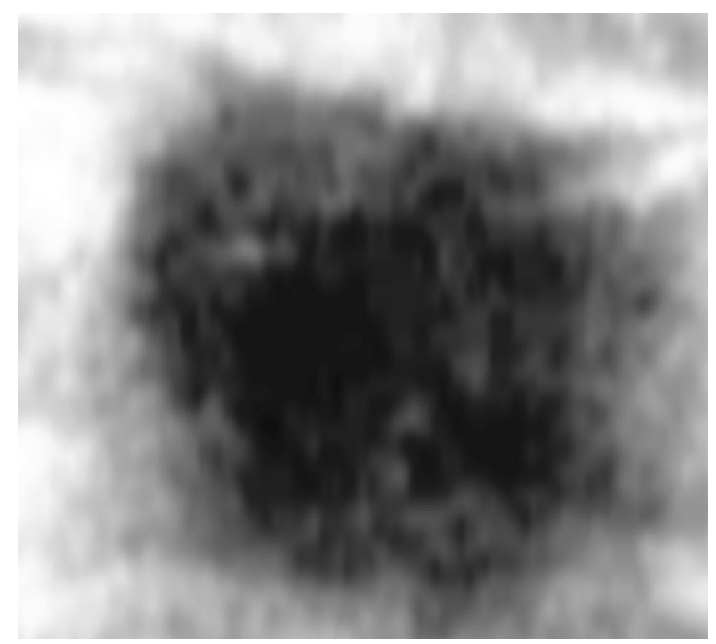

Figure 4. Enhanced ROI image.

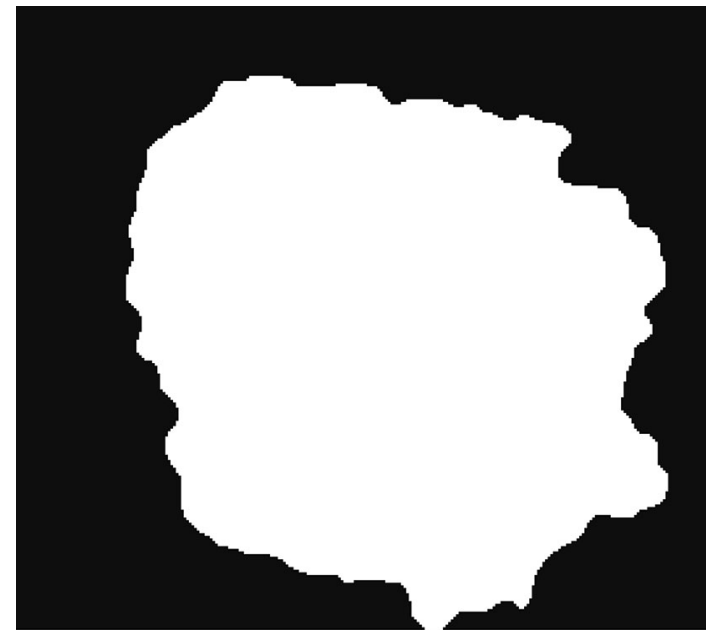

Figure 5. Binary form of ROI image.

contrast limited adaptive histogram equalization. Figures 4 and 5 give the filtered enhanced image and binary image.

\subsection{A novel texture-based feature set}

In this study, a total of eight statistical texture features (first-order mean obtained by the whole of the ROI (TF1), kurtosis (TF2), variance (TF3) and standard deviation (TF4) obtained via the approach mentioned in section 2.3 [10] and also the contrast (HF1), homogeneity (HF2), energy (HF3) and correlation (HF4) obtained via the second-order GLCM matrix) were divided to the tumour area in pixels, which is a morphological feature. Thus a novel texture-based feature set is obtained.

\subsection{Morphological features}

Five morphological features, namely roundness based on the BI-RADS lexicon and the expert doctor opinion (MF1), area (MF2), area/perimeter rate (MF3), long-/short-axis rate (MF4) and most importantly spiculation (MF5), were extracted. To get these features, first, the image was converted to black and white by applying Otsu global thresholding [69] to the filtered and enhanced image. Second, the shape of the mass in the ultrasound image was tried to better expose via the morphological open-close processes [70]. The binary image is shown in figure 5. The area in white colour represents the mass while the area in black colour represents the image background.The method based on the Fourier descriptors mentioned in section 2.4 was applied with an enhancement to calculate the spiculation feature. With this enhancement, an array is constructed by calculating the distances from two adjacent pixels of the mass edge to the centre pixel. Then the Fourier transform of this array is taken and the first value of the transformed data array is chosen as a feature. 


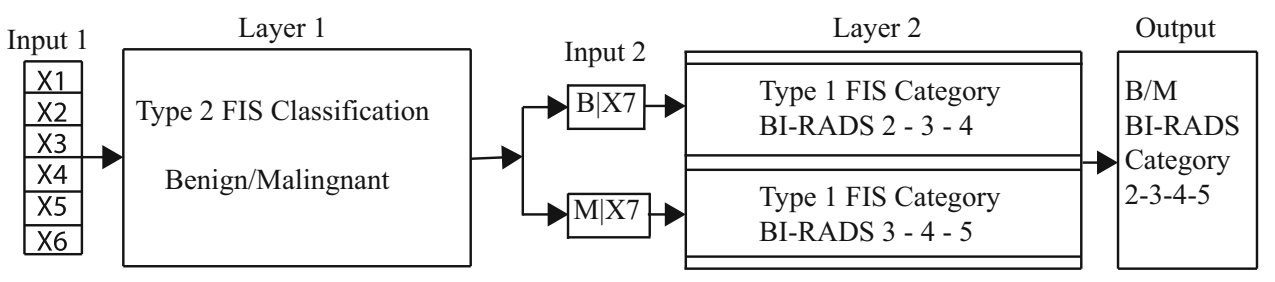

Figure 6. Proposed CAD architecture.

\subsection{Feature selection}

Five morphological features mentioned in BI-RADS lexicon (section 2.4) and in section 4.4 by the expert doctor $[71,72]$ were directly used. In the selection of texture features, the statistical method explained in section 2.5 was applied. First, the distribution over data set was investigated by applying Kolmogorov-Smirnov test via SPSS. Student's $t$-test was applied to the data set with normal distribution while Mann-Whitney $U$ test was applied to the data set without normal distribution. Subsequently, based on the $p$ value, the mean (TF1) and the contrast (HF1) were chosen as texture features $[73,74]$.

\subsection{Classification}

It is advisable to use BI-RADS lexicon in the decision process because it follows a natural language structure. The 'If the mass is rounded and less dense, then it is more probable to be classified as benign' rule can be conveniently modelled with fuzzy sets [75]. Adaptive neural fuzzy inference system (ANFIS) is a first-order, networkbased, multi-layer and adaptive Takagi-Sugeno fuzzy inference system proposed by Jang in 1993 [76, 77]. In a study the system architecture was designed, as each ANFIS output is an input to the next ANFIS layer [78]. In this model, a multi-layer classifier architecture based on Type-2 fuzzy inference system for the CAD system is proposed. Layer 1 classifies the mass as benign or malignant. The class at the output of Layer 1 enters the category classifier at Layer 2 where the BI-RADS category of the mass is determined. The architecture of Type-2 fuzzy inference system was initially designed as Type 1 for testing purposes using the MATLAB fuzzy logic toolbox. Then the FOU sizes were determined using $\mathrm{ABC}$ optimization algorithm and the system was converted to Type- 2 structure using type reduction module with the Karnik-Mendel algorithm $[79,80]$. A software has been developed in Matlab for every step. The block schema of the proposed architecture is shown in figure 6. Here, $X=X 1, X 2, X 3, X 4, X 5$, textitX6 is the Layer 1 input feature vector, $X 7$ is the Layer 2 input feature vector, $\mathrm{B} / \mathrm{M}$ is the benign/malignant output of Layer 1 and 2-5 are BI-RADS category output of Layer 2.Histograms were used in the design of each membership function [81]. The histograms of each feature data set in the benign and malignant classes were extracted and the most similar fuzzy set was determined as a triangular fuzzy set. For the consequent part (or output), again triangular fuzzy sets were preferred and they were scaled as $1-3$ for benign and 3-5 for malignant for the discrimination of classes. The histograms of benign and malignant data sets for $A p \_r a t i o$ feature are given as examples in figures 7 and 8 . The parameters used in the design of triangular membership functions were obtained by calculating the minimum, average and maximum values of feature data sets in each class and are given in table 2.

Mamdani-type rules were created via max-min approach by noting the medical information that the expert doctor uses during the interpretation of the ultrasound images [75]. The rules of the designed system are shown in table 3 .

The BI-RADS categories were determined as 2, 3, 4 for the benign tumour and 3, 4, 5 for the malignant tumour by the expert doctor. In this study, MF4 feature was used to classify the BI-RADS categories. The method used in Layer 1 was applied to the BI-RADS categories of each class and the input/output membership functions were designed. The parameter values of benign and malignant class input membership functions are given in tables 4 and 5 , respectively. The parameter values of output membership functions are given in table 6. The rules [82] of Layer 2 for

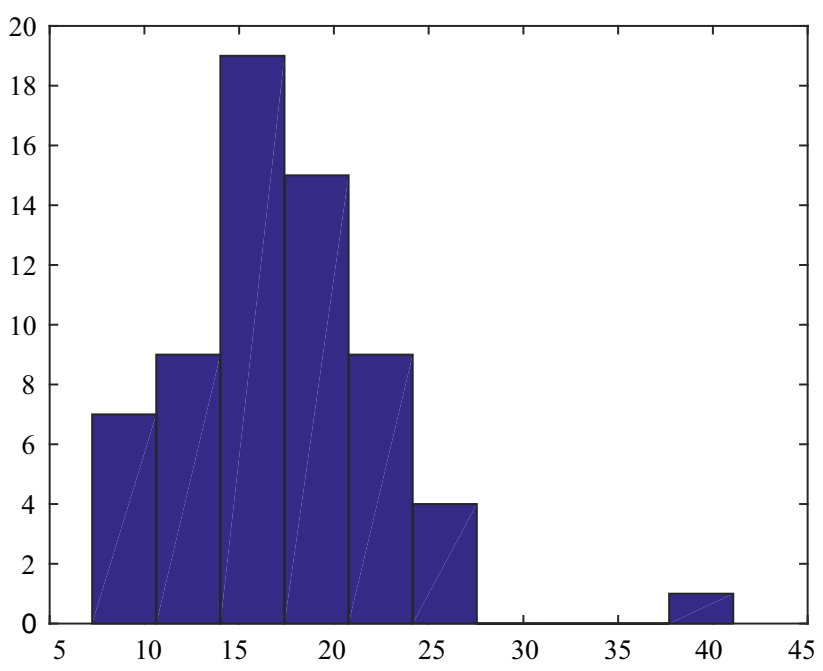

Figure 7. Histogram of benign data sets for Ap_ratio feature. 


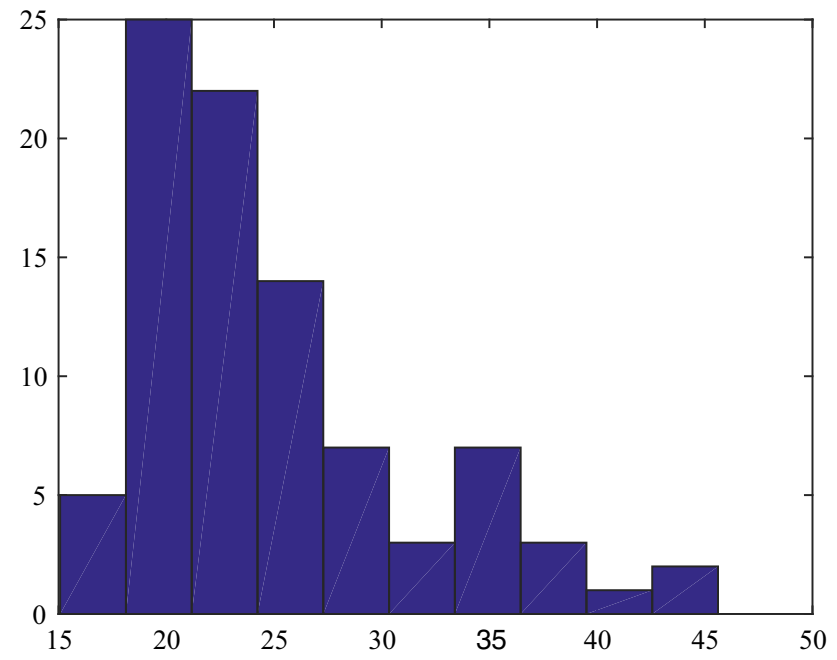

Figure 8. Histogram of malignant data sets for Ap_ratio feature.

Table 2. Minimum, mean and maximum values of the features.

\begin{tabular}{lccc}
\hline Feature & Min & Mean & Max \\
\hline MF5 B LOW & 143.120 & 451.430 & 568.830 \\
MF5 M HIGH & 572.750 & 613.470 & 685.840 \\
MF2 B LOW & 721 & 5439 & 9761 \\
MF2 M HIGH & 10022 & 15608 & 31927 \\
MF1 B LOW & 0.361 & 2.125 & 3.737 \\
MF1 M HIGH & 3.563 & 5.360 & 10.316 \\
MF3 B LOW & 7.240 & 16.270 & 25.620 \\
MF3 M HIGH & 14.780 & 37.250 & 83.230 \\
HF1 B HIGH & 15.330 & 84.610 & 706 \\
HF1 M LOW & 6.950 & 19.240 & 36 \\
TF1 B HIGH & 61.177 & 223.600 & 1206 \\
TF1 M LOW & 20.076 & 61.990 & 102.910 \\
\hline
\end{tabular}

Table 3. Layer 1 fuzzy rules of the CAD system.

\begin{tabular}{lc}
\hline Fuzzy rules & Class B/M \\
\hline If MF5 is high and MF2 is low & M \\
If MF5 is low and MF2 is high & $\mathrm{B}$ \\
If MF2 is high and HF1 is low & $\mathrm{M}$ \\
If MF2 is low and HF1 is high & $\mathrm{M}$ \\
If MF5 is high and MF3 is high & $\mathrm{B}$ \\
If MF5 is low and MF3 is low & $\mathrm{M}$ \\
If MF5 is high and HF1 is low & $\mathrm{B}$ \\
If MF5 is low and HF1 is high & $\mathrm{M}$ \\
If MF1 is high and TF1 is low & $\mathrm{B}$ \\
If MF1 is low and TF1 is high
\end{tabular}

the BI-RADS classification are given in table 7 . The membership functions that were initially designed as Type 1 constitute the upper membership functions in the conversion to Type 2 . The optimum FOU for each membership function was obtained using the ABC algorithm [83]. Thus,
Table 4. Parameter values for the benign class triangular membership function (min - minimum, max - maximum).

\begin{tabular}{lccc}
\hline Feature & Min & Mean & Max \\
\hline MF4 - BIRADS 2 - High & 1.16 & 2.50 & 3.38 \\
MF4 - BIRADS 3 - Middle & 1.23 & 1.74 & 2.20 \\
MF4 - BIRADS 4 - Low & 1.15 & 1.46 & 1.81 \\
\hline
\end{tabular}

Table 5. Parameter values for the malignant class triangular membership function (min - minimum, max - maximum).

\begin{tabular}{lccc}
\hline Feature & Min & Mean & Max \\
\hline MF4 - BIRADS 3 - High & 1.38 & 1.76 & 2.45 \\
MF4 - BIRADS 4 - Middle & 1.23 & 1.74 & 2.39 \\
MF4 - BIRADS 5 - Low & 1.02 & 1.31 & 1.86 \\
\hline
\end{tabular}

Table 6. Parameter values of output membership functions $\left(X_{1}, X_{3}, X_{5}\right.$ from figure 1$)$.

\begin{tabular}{lccc}
\hline BI-RADS class & $X_{1}$ & $X_{3}$ & $X_{5}$ \\
\hline Malignant BI-RADS 3 & 1 & 1.5 & 2 \\
Malignant BI-RADS 4 & 2 & 2.5 & 3 \\
Malignant BI-RADS 5 & 3 & 3.5 & 4 \\
Benign BI-RADS 2 & 1 & 1.5 & 2 \\
Benign BI-RADS 3 & 2 & 2.5 & 3 \\
Benign BI-RADS 4 & 3 & 3.5 & 4 \\
\hline
\end{tabular}

Table 7. Layer 2 fuzzy rules for benign/malignant class.

\begin{tabular}{lc}
\hline fuzzy rules & BI-RADS category \\
\hline If MF4 is high & BI-RADS 2 - Benign \\
If MF4 is middle & BI-RADS 3 - Benign \\
If MF4 is low & BI-RADS 4 - Benign \\
If MF4 is high & BI-RADS 3 - Malignant \\
If MF4 is middle & BI-RADS 4 - Malignant \\
If MF4 is low & BI-RADS 5 - Malignant \\
\hline
\end{tabular}

the parameter values of the lower membership function were calculated. The triangular membership function is given in figure 1. Here, $X_{1}, X_{3}$ and $X_{5}$ correspond to the minimum, mean and maximum values, respectively, for the given feature data set, $X_{2}=X_{1}+F O U$ and $X_{4}=X_{5}-F O U$. Optimal FOU sizes are given in table 8 .

\section{Success test}

Success test was realized by a comparison of the proposed method with the common classifiers such as SVM, ANN, KNN and Type-1 FIS on correctness, sensitivity and specificity measures. The result is given in table 9. The 
Table 8. FOU sizes of the input and output membership functions.

\begin{tabular}{lcccccc}
\hline MF1 & MF2 & MF3 & MF5 & TF1 & HF1 & Output \\
\hline 5 & 50 & 5 & 10 & 2 & 6 & 0.8 \\
\hline
\end{tabular}

Table 9. Comparison between the proposed method and other methods in terms of the success test parameters.

\begin{tabular}{cccc}
\hline Classifier & Acc $(\%)$ & Sens $(\%)$ & Spec $(\%)$ \\
\hline SVM & 97 & 98 & 95 \\
ANN(MLP) & 98 & 100 & 97 \\
KNN (k=8) & 92 & 98 & 84 \\
C-MEAN & 72 & 50 & 100 \\
FC-MEAN & 73 & 54 & 100 \\
LDA & 89 & 84 & 97 \\
TYPE 1 FUZZY & 96 & 97 & 95 \\
PROPOSED METHOD & 99.34 & 100 & 98.44 \\
\hline
\end{tabular}

correctness, specificity and sensitivity equations are as follows.

In Eqs. (25)-(27), TP stands for malignant classification in the case of malignant tumour, FN stands for benign classification in the case of malignant tumour, TN stands for benign classification in the case of benign tumour and FP stands for malignant classification in the case of benign tumour. In another CAD system that uses the same ultrasound image database with this study, benign/malignant classification was realized using an SVM classifier and a correctness of $95.7 \%$, sensitivity of $87.5 \%$ and specificity of $100 \%$ were gained [30]. In another study where the BIRADS category classification was realized using logistic regression, a correctness rate of $81 \%$ was acquired [3]. In this study, a correctness of $92 \%$ was obtained. It is observed that the proposed method is more successful when compared with these studies. The radiologists generally perform visual inspection followed by an invasive and painful procedure called biopsy for accurate diagnosis. However, the subjective way of classifying abnormal tissues in breast is an ineffective, tiresome and time-consuming job. Therefore, the proposed system provides an efficient and objective method of classifying breast tumours in ultrasound images. This system can improve the decision making and can be helpful in reducing the number of false diagnoses and biopsies. Medical costs will be reduced as well.

$$
\begin{aligned}
\operatorname{Accuracy}(\text { acc }) & =(T P+T N) /(T P+T N+F P+F N), \\
\text { specificity }(\text { spec }) & =T N /(T N+F P), \\
\text { sensitivity }(\text { sens }) & =T P /(T P+F N) .
\end{aligned}
$$

\section{Conclusion and related work}

There are many studies in the literature that utilize CAD systems with various architectures to help the expert doctors in diagnosis stage. They include the artificial intelligence techniques that are benefited by the superior calculation ability of the computers. The proposed design is a high-success-rate, novel CAD architecture that combines the expert doctor knowledge with the superior calculation ability of the computers and it provides more accurate information to the doctor, including the BI-RADS category of the breast cancer. In the future, conversion of the designed CAD system to a web application is planned. In the future, the researchers who study in the field of this area can save verbal expressions that contain the knowledge and experiences of doctors' diagnosis to a database with a software module that is added to web-based application. Through the analysis of the verbal expression, it is possible to improve the rule structure of fuzzy system. Through this web-based application, doctors who are even far away from each other procure the chance of sharing the interpretations on patients while analysing images of patients in real time. By this way, the accuracy of diagnosis with a CAD system is increased. Since the application provides for sharing information and experiences, the application software, which is improved by the continuous studies, can be additionally used for educational purposes. One of the advantage of web-based CAD system is that all doctors who work in this field can easily have an access to this system via internet. Furthermore, monitoring the patient by the doctor is provided for without the limitations of time and location.

\section{Acknowledgements}

This study is supported by Erciyes University Scientific Research Projects unit with the ID of 6629. The authors are gratefull to Dr T S A Geertsma for supplying the ultrasound image database and Dr Bilge Oztoprak (Cumhuriyet University, Faculty of Medicine, Radiology Department) for commenting on ultrasound images medically.

\section{References}

[1] Dhahbi S, Barhoumi W and Zagrouba E 2015 Breast cancer diagnosis in digitized mammograms using curvelet moments. Computer in Biology and Medicine 64: 79-90

[2] Dora L, Agraval S, Panda R and Abraham A 2017 Optimal breast cancer classification using Gauss-Newton representation based algorithm. Expert Systems with Applications 85: 134-145

[3] Lo C M, Lai Y C, Chou Y H and Chang R F 2015 Quantitative breast lesion classification based on multichannel distributions in shear-wave imaging. Computer Methods and Programs in Biomedicine 122: 354-361 
[4] Reidt J M 1959 Diagnostic applications of ultrasound. Proceedings of the IRE 47: 1963-1967

[5] Shi X, Cheng H D, Hu L, Ju W and Tian J 2010 Detection and classification of masses in breast ultrasound images. Digital Signal Processing 20: 824-836.

[6] Stines J 2007 BI-RADS: Use in the French radiologic community. How to overcome with some difficulties. European Journal of Radiology 61: 224-234

[7] Hong A S, Rosen E L, Soo M S and Baker J 2005 BI-RADS for sonography: positive sonographic features. American Journal of Roentgenology 184: 1260-1265

[8] Park S, Shin D K and Kim J S 2013 Components of computer-aided diagnosis for breast ultrasound. IT Convergence Practice (INPRA) 1: 50-63

[9] Liu B, Cheng H D, Huang J, Tian J, Tang X and Liu J 2010 Fully automatic and segmentation-robust classification of breast tumors based on local texture analysis of ultrasound images. Pattern Recognition 43: 280-298

[10] Cheng H D, Shan J, Ju W, Guo Y and Zhang L 2010 Automated breast cancer detection and classification using ultrasound images: a survey. Pattern Recognition 43: 299-317

[11] Stoitsis J, Valavanis I, Mougiakakou S G, Golemati S, Nikita A and Nikita K S 2006 Computer aided diagnosis based on medical image processing and artificial intelligence methods. Nuclear Instruments and Methods in Physics Research 569: 591-595

[12] Sampaio W B, Diniz E M, Silva A C, Paiva A C and Gattass M 2011 Detection of masses in mammogram images using CNN, geostatistic functions and SVM. Computers in Biology and Medicine 41: 653-664

[13] Chiou H J, Chen C Y, Liu T C, Chiou S Y, Wang H K, Chou Y H and Chiang H K 2009 Computer-aided diagnosis of peripheral soft tissue masses based on ultrasound imaging. Computerized Medical Imaging and Graphics 33: 408-413

[14] Prabusankarlal K M, Manavalan R and Sivaranjani R 2017 An optimized non local means filter using automated clustering based preclassification through gap statistics for speckle reduction in breast ultrasound images. Applied Computing and Informatics 14(1): 48-54

[15] Deka B and Bora P K 2013 Removal of correlated speckle noise using sparse and overcomplete representations. Biomedical Signal Processing and Control 8: 520-533

[16] Latifoglu F 2013 A novel approach to speckle noise filtering based on Artificial Bee Colony algorithm: an ultrasound image application. Computer Methods and Programs in Biomedicine 111: 561-569

[17] Guo Y, Wang Y and Hou T 2011 Speckle filtering of ultrasonic images using a modified non local-based algorithm. Biomedical Signal Processing and Control 6: 129-138

[18] Shan J 2011 A fully automatic segmentation method for breast ultrasound images. Mphil Thesis, Utah State University, Logan, Utah

[19] Sellami L, Sassi O B, Chtourou K and Hamida A B 2015 Breast cancer ultrasound images sequence exploration using BI-RADS features extraction: towards an advanced clinical aided tool for precise lesion characterization. IEEE Transactions on Nanobioscience 14: 740-745

[20] Huang Y L, Wang K L and Chen D R 2006 Diagnosis of breast tumors with ultrasonic texture analysis using support vector machines. Neural Computing and Applications 15: 164-169

[21] Zhou S, Shi J, Zhu J, Cai Y and Wang R 2013 Shearlet-based texture feature extraction for classification of breast tumor in ultrasound image. Biomedical Signal Processing and Control 8: 688-696

[22] Nasser M A, Melendez J, Moreno A, Omer O A and Puig D 2017 Breast tumor classification in ultrasound images using texture analysis and super-resolution methods. Engineering Applications of Artificial Intelligence 59: 84-92

[23] Xian G M 2010 An identification method of malignant and benign liver tumors from ultrasonography based on GLCM texture features and fuzzy SVM. Expert Systems with Applications 37: 6737-6741

[24] Gonzales R and Woods R E 1992 Digital image processing, 2nd ed. Upper Saddle River, New Jersey 07458, USA: Prentice-Hall

[25] Ondimu S N and Murase H 2008 Effect of probability-distance based Markovian texture extraction on discrimination in biological imaging. Computers and Electronics in Agriculture 63: 2-12

[26] Haralick R M, Shanmugam K and Dinstein I 1973 Textural features for image classification. IEEE Transactions on Systems, Man, and Cybernetics 3(6): 610-621

[27] Materka A and Strzelecki M 1998 Texture analysis methods - a review. Tecnical University of Lodz, Institute of Electronics, COST B11 Report, Brussels

[28] Amin K M, Shahin A I and Guo Y 2016 A novel breast tumor classification algorithm using neutrosophic score features. Measurement 81: 210-220

[29] Chang R F, Wu W J, Moon W K and Chen D R 2005 Automatic ultrasound segmentation and morphology based diagnosis of solid breast tumors. Breast Cancer Research and Treatment 89: 179-185

[30] Menon R V, Raha P, Kothari S, Chakraborty, Chakrabarti I and Karim R 2015 Automated detection and classification of mass from breast ultrasound images. In: Proceedings of the NCVPRIPG Conference, 16-19 December, Patna, India, pp. 3-6

[31] Chen D R, Chien C L and Kuo Y F 2015 Computer-aided assessment of tumor grade for breast cancer in ultrasound images. Computational and Mathematical Methods in Medicine ID 914091, 6 pp.

[32] Chen Y and Huang Q 2016 An approach based on biclustering and neural network for classification of lesions in breast ultrasound. In: Proceedings of the International Conference on Advanced Robotics and Mechatronics, 18-20 August 2016, Macau, China, pp. 597-601

[33] Singh B K, Verma K and Thoke A S 2016 Fuzzy cluster based neural network classifier for classifying breast tumors in ultrasound images. Expert Systems with Applications 66: 114-123

[34] Wu W J, Lin S W and Moon W K 2012 Combining support vector machine with genetic algorithm to classify ultrasound breast tumor images. Computerized Medical Imaging and Graphics 36: 627-633

[35] Liao R, Wan T and Qin Z 2011 Classification of benign and malignant breast tumors in ultrasound images based on multiple sonographic and textural features. In: Proceedings of the Third International Conference on Intelligent Human- 
Machine Systems and Cybernetics, 10 October 2011, Zhejiang, China, pp. 71-74

[36] Singh B K, Verma K, Thoke A S and Suri J S 2017 Risk stratification of 2D ultrasound-based breast lesions using hybrid feature selection in machine learning paradigm. Measurement 105: 146-157

[37] Lee H and Chen Y P P 2015 Image based computer aided diagnosis system for cancer detection. Expert Systems with Applications 42: 5356-5365

[38] Prabusankarlal K M, Thirumoorthy P and Manavalan R 2015 Assessment of combined textural and morphological features for diagnosis of breast masses in ultrasound. Human-centric Computing and Information Sciences 5: 12

[39] Alvarenga A V, Infantosi A F C, Pereira W C A and Azevedo C M 2010 Assessing the performance of morphological parameters in distinguishing breast tumors on ultrasound images. Medical Engineering \& Physics 32: 49-56

[40] Minavathi, Murali S and Dinesh M S 2012 Classification of mass in breast ultrasound images using image processing techniques. International Journal of Computer Applications 42: $29-36$.

[41] Saranya P K and Samundeeswari E S 2016 A study on morphological and textural features for classifying breast lesion. International Journal of Innovative Research in Science, Engineering and Technology 5: 3267-3279

[42] Nayeem M A R, Joadder A M, Shetu S A, Jamil F R and Helal A A 2014 Feature selection for breast cancer detection from ultrasound images. In: Proceedings of the International Conference on Informatics, Electronics \& Vision (ICIEV), 23-24 May 2014, Dhaka, Bangladesh, pp. 1-6

[43] Raza S, Goldkamp A L, Chikarmane S and Birdwell R L 2010 US of breast masses categorized as BI-RADS 3, 4, and 5: pictorial review of factors influencing clinical management. Radiographics 30: 1199-1213

[44] Huang Y L, Chen D R, Jiang Y R, Kuo S J, Wu H K and Moon W K 2008 Computer-aided diagnosis using morphological features for classifying breast lesions on ultrasound. Ultrasound in Obstetrics and Gynecology 32: 565-572

[45] George Y M, Zayed H H, Roushdy M I and Elbagoury B M 2014 Remote computer-aided breast cancer detection and diagnosis system based on cytological images. IEEE Systems Journal 8: 949-964

[46] Gonzaga M A 2010 How accurate is ultrasound in evaluating palpable breast masses? Pan African Medical Journal 7: 1937-8688

[47] Tan T, Platel B, Huisman B H, Sanchez C I, Mus R and Karssemeijer N. Computer-aided lesion diagnosis in automated 3-D breast ultrasound using coronal spiculation. IEEE Transactions on Medical Imaging 31: 1034-1042

[48] Rangayyan R M, Mudigonda N R and Desautels J E L 2000 Boundary modelling and shape analysis methods for classification of mammographic masses. Medical \& Biological Engineering \& Computing 38: 487-496

[49] Shen L, Rangayyan R M and Desautels J E L 1994 Application of shape analysis to mammographic calcifications. IEEE Transactions on Medical Imaging 13: 263-274

[50] Joo S, Moon W K and Kim H C 2004 Computer-aided diagnosis of solid breast nodules: use of an artificial neural network based on multiple sonographic features. IEEE Transactions on Medical Imaging 23: 1292-1300
[51] Barbucha D, Nguyen N T and Batubara J 2015 New trends in intelligent information and database systems. In: Verma K, Singh B K, Tripathi P and Thoke A S (Eds). Review of feature selection algorithms for breast cancer ultrasound image. Switzerland: Springer International Publishing, 2015, pp. 23-32

[52] Azar A T and El-Said S A 2013 Probabilistic neural network for breast cancer classification. Neural Computing and Applications 23: 1737-1751

[53] Moon W K, et al 2017 Computer-aided tumor diagnosis using shear wave breast elastography. Ultrasonics 78: $125-133$

[54] Chen D R, Chang R F, Chen J C, Ho M F, Kuo S J, Chen S T, Hung S J and Moon W K 2005 Classification of breast ultrasound images using fractal feature. Journal of Clinical Imaging 29: 235-245

[55] Acharya U R, Ng W L, Rahmat K, Sudarshan V K, Koh J E W, Tan J H, Yeong C H and Ng K H 2017 Data mining framework for breast lesion classification in shear wave ultrasound: a hybrid feature paradigm. Biomedical Signal Processing and Control 33: 400-410

[56] Ding J, Cheng H D, Xian M, Zhang Y and Xu F 2015 Localweighted citation- $\mathrm{kNN}$ algorithm for breast ultrasound image classification. Optik 126: 5188-5193

[57] Zadeh L A 1975 Fuzzy logic and approximate reasoning. Synthese 30: 407-428

[58] Mamdani E H 1974 Application of fuzzy algorithms for control of simple dynamic plant. Proceedings of the Institution of Electrical Engineers 121: 1585-1588

[59] Choi B I, Chung F and Rhee H 2009 Interval type-2 fuzzy membership function generation methods for pattern recognition. Information Sciences 179: 2102-2122

[60] Akgun A, Sezer E A, Nefeslioglu H A, Gokceoglu C and Pradhan B 2012 An easy-to-use MATLAB program (MamLand) for the assessment of landslide susceptibility using a Mamdani fuzzy algorithm. Computers and Geosciences 38 : 23-34

[61] Mendel J M 2007 Type-2 fuzzy sets and systems: an overview. IEEE Computational Intelligence Magazine 2: 20-29

[62] Castillo O and Melin P 2012 Type-2 fuzzy logic systems. In: Recent advances in interval type-2 fuzzy systems. Heidelberg-New York-Dordrecht-London: Springer

[63] Hosseini R, Qanadli S D, Barman S, Mazinani M, Ellis T and Dehmeshki J 2012 An automatic approach for learning and tuning Gaussian interval type-2 fuzzy membership functions applied to lung CAD classification system. IEEE Transactions on Fuzzy Systems 20: 224-234

[64] Juang C F, Huang R B and Lin Y Y 2009 A recurrent selfevolving interval type-2 fuzzy neural network for dynamic system processing. IEEE Transactions on Fuzzy Systems 17: 1092-1105

[65] Zhai D and Mendel J M 2011 Uncertainty measures for general Type-2 fuzzy sets. Information Sciences 181: 503-518

[66] Mendel J M, John R I and Liu F 2006 Interval type-2 fuzzy logic systems made simple. IEEE Transactions on Fuzzy Systems 14: 808-821

[67] Hidalgo D, Melin P and Castillo O 2012 An optimization method for designing type-2 fuzzy inference systems based on the footprint of uncertainty using genetic algorithms. Expert Systems with Applications 39: 4590-4598 
[68] Raffei A F M, Asmuni H, Hassan R and Othman R M. A low lighting or contrast ratio visible iris recognition using isocontrast limited adaptive histogram equalization. Knowledge-Based Systems 74: 40-48

[69] Sha C, Hou J and Cui H 2016 A robust 2D Otsu's thresholding method in image segmentation. Journal of Visual Communication and Image Representation. 41: 339-351

[70] Gomez W, Leija L, Pereira W C A and Infantosi A F C 2009 Morphological operators on the segmentation of breast ultrasound images. In: Proceedings of the Pan American Health Care Exchanges - PAHCE, 16-20 March 2009, Mexico City, Mexico, pp. 67-71

[71] Moon W K, Lo M C, Cho N, Chang J M, Huang C S, Chen J $\mathrm{H}$ and Chang R F 2013 Computer-aided diagnosis of breast masses using quantified BI-RADS findings. Computer Methods and Programs in Biomedicine 111: 84-92

[72] Giger M L 2000 Computer-aided diagnosis of breast lesions in medical images. Computing in Medicine 58: 39-45

[73] Lo C M, Chang Y C, Yang Y W, Huang C S and Chang R F 2015 Quantitative breast mass classification based on the integration of B-mode features and strain features in elastography. Computers in Biology and Medicine 64: 91-100

[74] Moon W K, Lo C M, Chang J M, Huang C S, Chen J H, and Chang R F 2013 Quantitative Ultrasound Analysis for Classification of BI-RADS Category 3 Breast Masses. Journal of Digital Imaging 26: 1091-1098

[75] Miranda G H B and Felipe J C 2015 Computer-aided diagnosis system based on fuzzy logic for breast cancer categorization. Computers in Biology and Medicine 64: 334-346

[76] You H, Ma Z, Tang Y, Wang Y, Yan J, Cen K, Ni M and Huang Q 2017 Comparison of ANN (MLP), ANFIS, SVM, and RF models for the online classification of heating value of burning municipal solid waste in circulating fluidized bed incinerators. Waste Management 68: 186-197
[77] Mousa R, Munib Q and Moussa A 2005 Breast cancer diagnosis system based on wavelet analysis and fuzzy-neural. Expert Systems with Applications 28: 713-723

[78] Iraji M S 2017 Multi-layer architecture for adaptive fuzzy inference system with a large number of input features. Cognitive Systems Research 42: 23-41

[79] Mendel J M 2013 On KM algorithms for solving type-2 fuzzy set problems. IEEE Transactions on Fuzzy Systems 21: 426-446

[80] Wu D and Mendel J M 2014 Designing practical interval type-2 fuzzy logic systems made simple. In: Proceedings of the IEEE International Conference on Fuzzy Systems (FUZZIEEE), 6-11 July 2014, Beijing, China, pp. 800-807

[81] Chumklin S, Auephanwiriyakul S and Umpon N T 2010 Microcalcification detection in mammograms using interval type-2 fuzzy logic system with automatic membership function generation. In: Proceedings of Fuzzy Systems (FUZZ), 2010 IEEE International Conference, 18-23 July 2010, Barcelona, Spain

[82] Treesatayapun C and Uatrongjit S 2005 Adaptive controller with fuzzy rules emulated structure and its applications. Engineering Applications of Artificial Intelligence 18: 603-615

[83] Nozohour-Leilabady B N and Fazelabdolabadi B 2016 On the application of artificial bee colony (ABC) algorithm for optimization of well placements in fractured reservoirs; efficiency comparison with the particle swarm optimization (PSO) methodology. Petroleum 2: 79-89

[84] Lin C, Hou Y, Chen T and Chen K 2014 Breast nodules computer-aided diagnostic system design using fuzzy cerebellar model neural networks. IEEE Transactions on Fuzzy Systems 22: 693-699 